\title{
Steady Vortex Breakdown in Swirling Flow Inside a Closed Container: Numerical Simulations, PIV and LDV Measurements
}

\author{
J.M.M. Sousa* \\ Department of Mechanical Engineering, Instituto Superior Técnico, Technical University of Lisbon, Av. Rovisco Pais, \\ 1049-001 Lisboa, Portugal
}

\begin{abstract}
Numerical simulations and measurements using PIV and LDV have been carried out for steady vortex breakdown inside a closed container. The numerical solution has been employed as a benchmark to assess the capabilities and limitations of the experimental techniques in a difficult flow case involving a double breakdown in the limit of the steady regime.
\end{abstract}

\section{INTRODUCTION}

The swirling flow inside a closed container with a rotating end-wall has attracted significant interest from researchers since more than three decades. The simplicity of the geometry, the well-controlled flow conditions, the highly rich physics of the associated phenomena and, especially, the importance of related applications certainly explain the attention given. Initial studies on this matter were attributed to Vogel [1] but the experiments of Escudier [2], published sixteen years later, were the first reporting a systematic investigation on the occurrence of vortex breakdown in this flow geometry.

Since then, aiming to improve our knowledge about this problem, many experiments and numerical simulations have been carried out for a wide range of flow conditions. Recently, the majority of these studies have employed numerical simulations to describe the onset of unsteady, asymmetric behaviour of vortex breakdown structures [3-8]. On the other hand, and with a few exceptions only [9-12], the experimental investigations on this subject available in the literature are essentially based on the results of flow visualization techniques. In addition, it would be expected that before embarking on detailed analyses of a more complicated behaviour, such as that associated to unsteadiness and symmetry-breaking, a thorough assessment of the performance of quantitative experimental techniques should be made in the context of steady, symmetric breakdown.

In the present study, the variant proposed by Pereira and Sousa [10] to drive the swirling flow using a rotating cone instead of a flat lid has been considered with the purpose of accomplishing the foregoing task. This also differs from the numerical investigations by $\mathrm{Yu}$ et al. [13], who used stationary (concave and convex) conical lids. The velocity field in a meridional plane of the closed container has been measured by the use of both Particle Image Velocimetry

*Address correspondence to this author at the Department of Mechanical Engineering, Instituto Superior Técnico, Technical University of Lisbon, Av. Rovisco Pais, 1049001 Lisboa, Portugal; E-mail: msousa@ist.utl.pt
(PIV) and Laser-Doppler Velocimetry (LDV), for a particular flow condition displaying steady, double breakdown of the columnar vortex at the axis. Numerical simulations have also been carried out to serve as a benchmark with the aim of assessing the capabilities and limitations of the aforementioned experimental techniques.

\section{MATERIALS AND METHODOLOGY}

The swirling flow inside the closed container was generated by the rotation of a conical end-wall at a constant value of the angular speed $\Omega$, as shown in Fig. (1). The main idea behind the use of a conical driver instead of the classical flat rotor is that, from a topological point of view, the apex of a cone provides a well-defined nodal point for the central streamline impinging upon the rotor wall. The container was a cylinder with radius $R$ and height $H=3 R$, which was kept unchanged in this study.

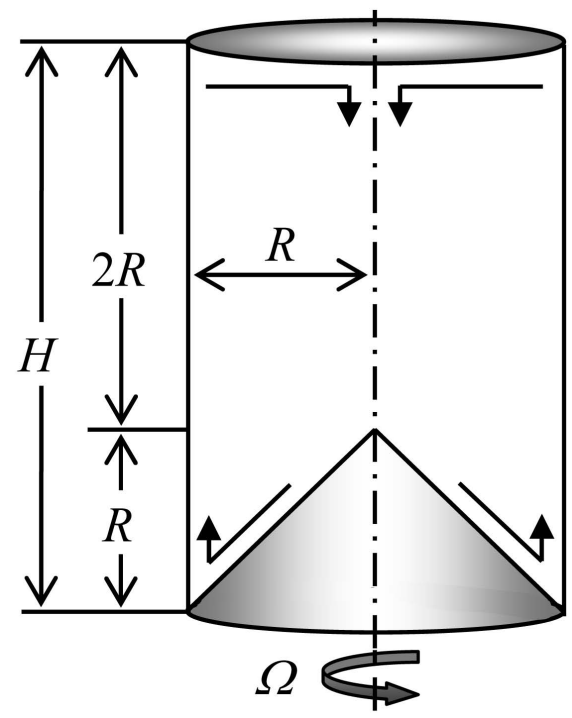

Fig. (1). Geometry of the closed container with a rotating conical end-wall.

The flow condition is defined by the pair of parameters ( $R e, H / R$ ), where $R e=\Omega R^{2} / v=2570$ is the Reynolds number and $v$ is the kinematic viscosity of the fluid. The stability 
diagram in Fig. (2) indicates whether single, double or no vortex breakdown occurs as well as if the flow regime is steady or time-dependent [10]. In this case, a steady, double breakdown structure is expected to be observed.

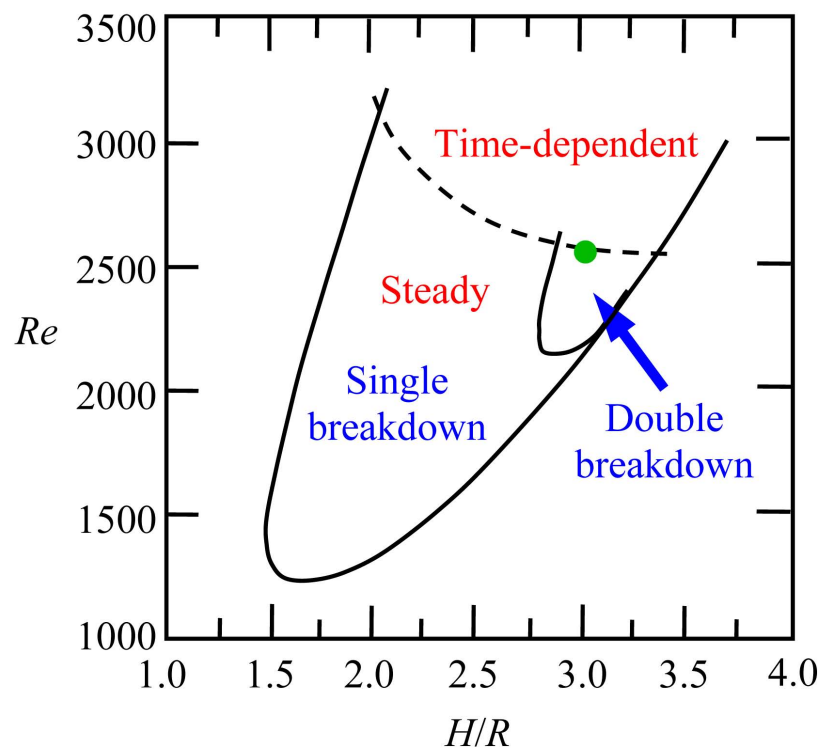

Fig. (2). Stability diagram [10] (the green spot marks the investigated flow condition).

Numerical simulations and measurements employing both PIV and LDV have been carried out for the aforementioned flow condition. A synthetic oil $\left(v=31.2 \times 10^{-6} \mathrm{~m}^{2} \mathrm{~s}^{-1}\right.$ @ $21^{\circ} \mathrm{C}$ ) was used as working fluid in the experiments in order to allow refractive index matching between the container walls and the fluid, as depicted in Fig. (3). For this purpose, the oil temperature was controlled with an accuracy of $\pm 0.1{ }^{\circ} \mathrm{C}$, which further allowed to minimize the uncertainty in $R e$. Additional details concerning the experimental setup were given in [10].

\section{NUMERICAL SIMULATIONS}

The mass and momentum conservation equations for the unsteady, three-dimensional flow of an incompressible fluid have been solved using the finite-volume methodology. These were discretized on a structured, non-orthogonal, fine mesh comprising 122 × $82 \times 82$ nodes, and integrated in space over each control volume as well as in time over a small time interval $\Delta t$. The Crank-Nicholson time-stepping method was used to advance diffusion terms in time, whereas the second-order accurate Adams-Bashforth method was applied to convection terms. The time increment was determined by stability requirements, namely $\max (C F L) \leq$ 0.4, where $C F L$ stands for the Courant-Friedrichs-Lewy number. On the other hand, the spatial discretization of convection and diffusion terms was carried out employing second-order accurate central differences. Finally, the pressure field was obtained by an iterative procedure from the solution of a Poisson equation resulting from the combination of continuity and momentum equations. Additional details about the numerical methodology were given in [10].

A very long simulation time was required to obtain a virtually steady solution for the present values of $R e$ and $H / R$, namely $\Omega t>2000$. This is due to the fact that this pair of parameters is deliberately located on the boundary separating steady and unsteady regimes in the stability diagram shown in Fig. (2). Such flow condition was selected with the aim of emphasizing the occurrence of vortex breakdown as well as maximizing the associated field gradients.

\section{PIV MEASUREMENTS}

The digital implementation of PIV proposed in [14] was employed to collect a total of 300 flow images, which were used in the computation of ensemble-averaged velocity vectors. The associated statistical error in mean values is smaller than $2 \%$ for a $95 \%$ confidence level [15]. Following the conclusions of a previous study [16], the images have been processed in interrogation windows of $32 \times 32$ pixels with a $75 \%$ overlap.

In general, the dominant velocity component in swirling flows confined to closed containers is the azimuthal component. Thus, the main obstacle to an efficient application of PIV to these flows lies in its strong threedimensionality. Such large amount of motion normal to the light sheet leads to a very significant out-of-plane loss of particle pairs, which ultimately originates an important decrease in correlation peak detectability. There is no panacea for this problem, but at least three methods have been suggested for the minimization of the foregoing effects [17]. The present work combined both the reduction of the laser pulse delay ( $3 \mathrm{~ms}$ ) and the thickening of the light sheet (2.5 mm). A circular offset of the light sheet could also have been implemented at the expense of the system's simplicity. However, the efficacy of the latter procedure is rather questionable in this case due to large variations in the magnitude of the out-of-plane flow component. It must be mentioned that the laser pulse delay was further limited by exigent requirements in dynamic range, as a result of the broad range of velocities encountered in the presence of vortex breakdown. Additionally, the thickness of the light sheet had to be optimised taking into account that a reduction in illumination intensity also produces a decrease in signalto-noise ratio.

Wide variations in the velocity field translate into a large variability in particle image shift. Thus, higher velocities occurring around the vortex breakdown structures and near the container walls are particularly vulnerable to random and bias errors due to in-plane loss of particle pairs, mainly resulting in increased noise and underestimation of displacement values by the PIV system, respectively. In order to minimise the consequences of these effects, a discrete offset of the interrogation windows coupled with a multi-grid/pass procedure was applied as well [17]. This also allowed to further increase the dynamic range in the current arrangement. It will be shown later that this issue is particularly critical when whole-field measurements of the flow inside the closed container are performed using PIV.

\section{LDV MEASUREMENTS}

A two-component LDV system from TSI Inc. was also used in the experimental characterisation of the flow structures, although only measurements of the axial velocity component are presented here. The velocimeter was based on fiberoptics and operated in backward-scatter mode. Signal 


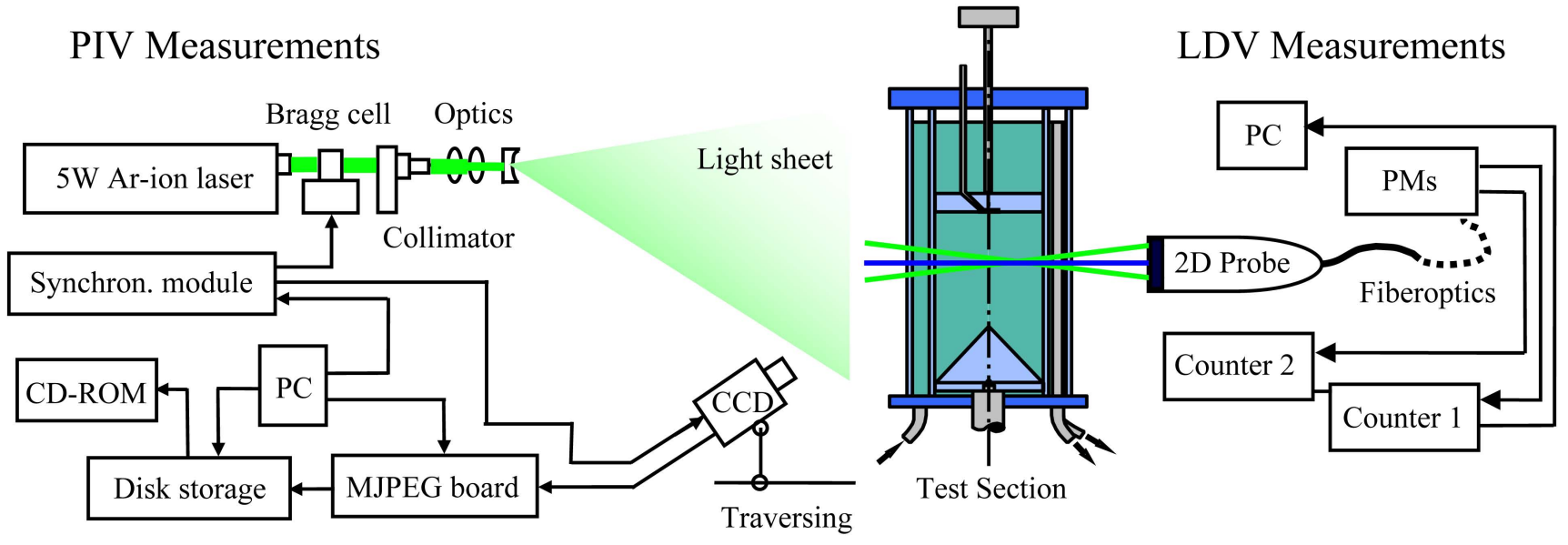

Fig. (3). Schematics of the experimental apparatus for PIV and LDV measurements.

processing was carried out using frequency counters. Velocity values were evaluated by ensemble-averaging, calculated from 5000 samples. The associated statistical error in mean values is smaller than $1 \%$ for a $95 \%$ confidence level [15]. Additional details regarding the system and the measuring effort can be found in [10].

The aforementioned refractive index matching procedure allowed to minimise the problem of distortion of the optical paths through the curved surfaces in the liquid flow [18], so that reliable measurements close to the container walls could be performed. Other systematic errors such as non-turbulent Doppler broadening due to gradients of mean velocity across the measuring volume and sampling bias are, in general, the prevalent sources of uncertainty in LDV measurements. However, the latter was lessened by the use of high seeding rates compared to the fundamental velocity fluctuation rates, and the former affects essentially the variances and other higher-order statistics of the velocity time series [15].

\section{RESULTS AND DISCUSSION}

The results obtained from PIV and LDV measurements are compared here with those produced by numerical simulation, which is used as a benchmark. This will allow a discussion on the limitations of each of these experimental techniques for a detailed characterisation of vortex breakdown and the remainder of the swirling flow inside the closed container. Taking into account that, for the investigated condition, the flow is steady and axisymmetric, only results in a meridional plane are presented. It must be emphasized that the measurement of the radial and, especially, the axial velocity components is more demanding than that of the azimuthal velocity field. Hence, the discussion is focused on the former only.

Fig. (4) shows the velocity field measured in a meridional plane of the closed container using PIV. The depicted flow topology strongly resembles that obtained by

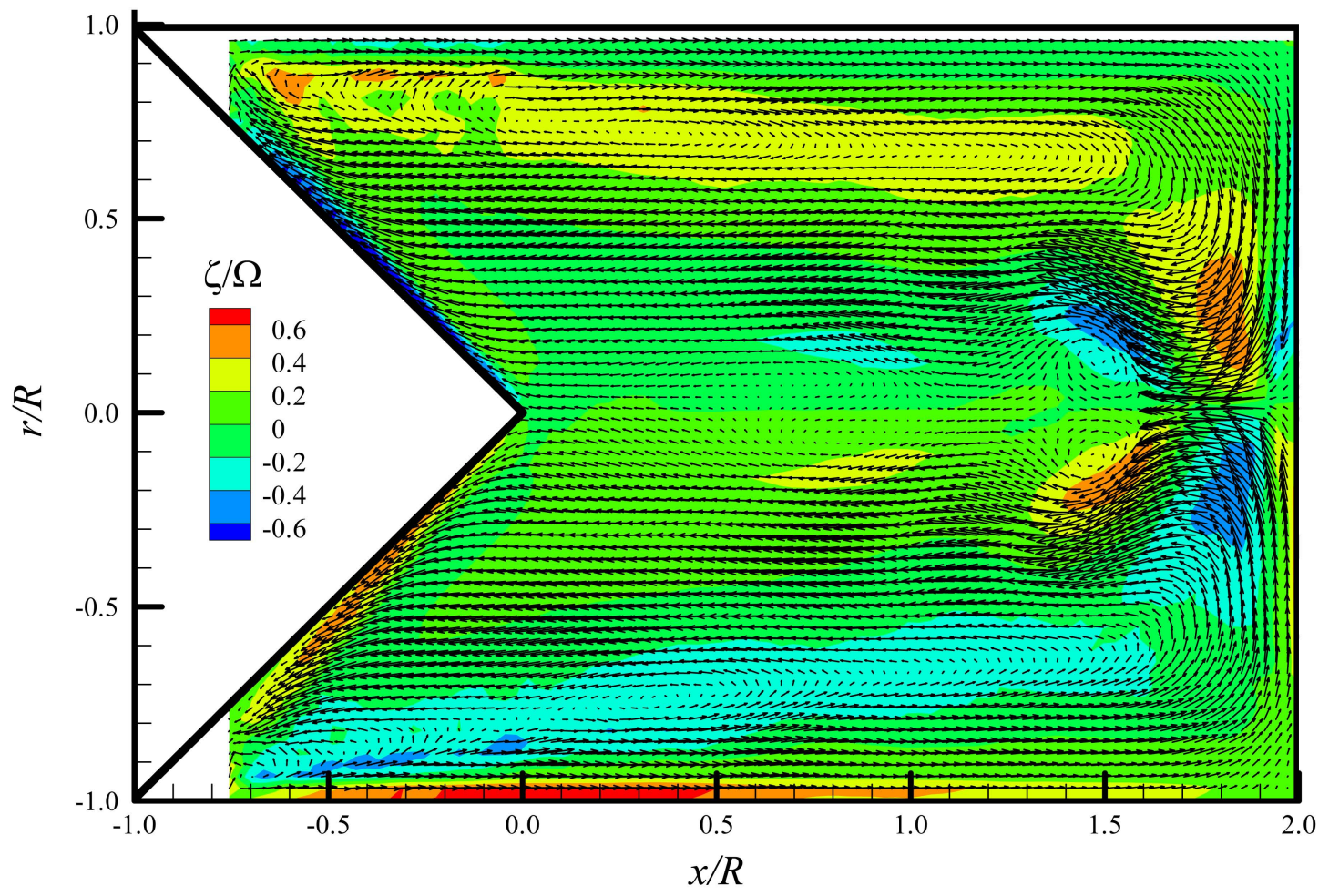

Fig. (4). Velocity vectors and vorticity contours in a meridional plane obtained by PIV measurements. 


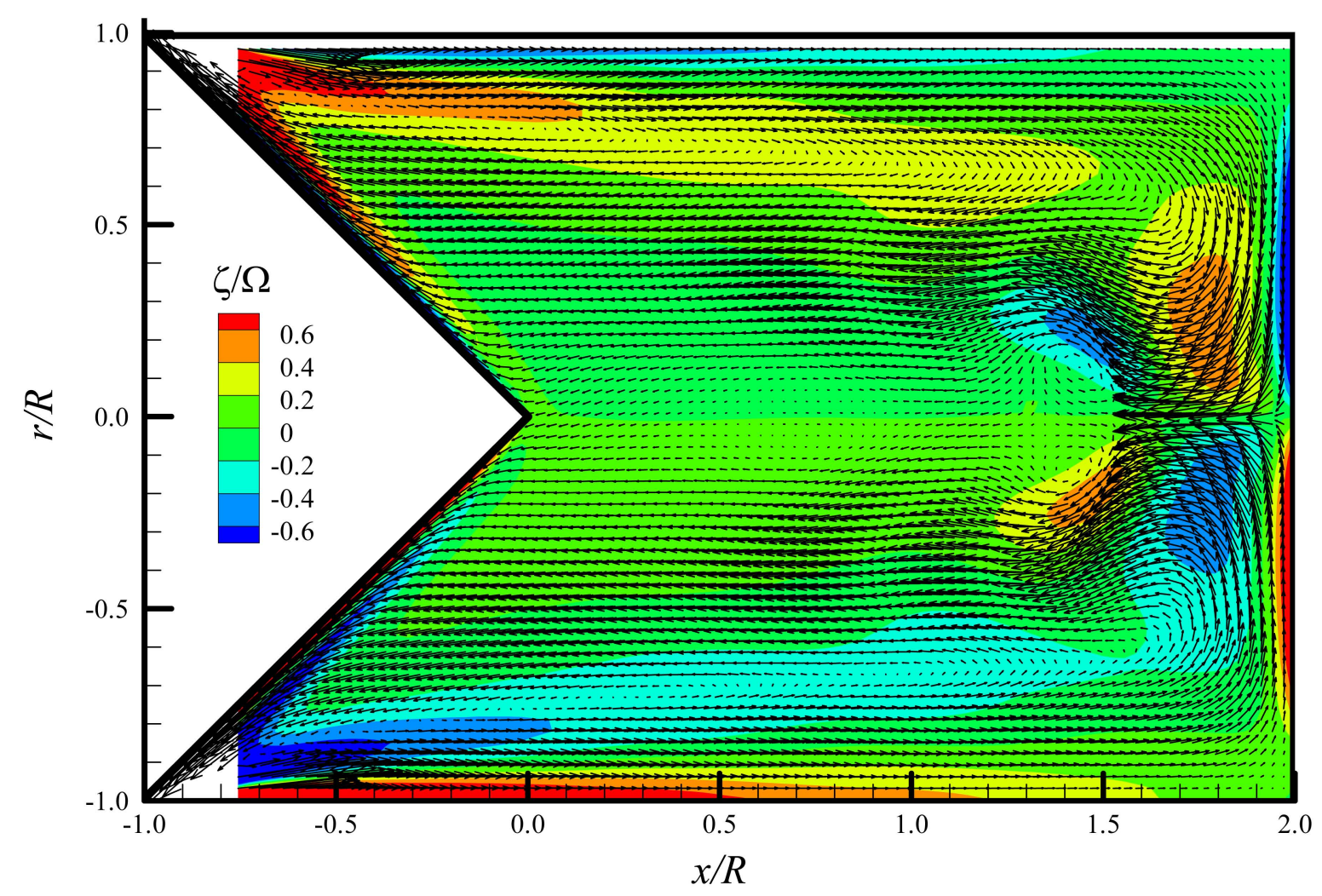

Fig. (5). Velocity vectors and vorticity contours in a meridional plane obtained by numerical simulation (as interpolated to the uniform mesh of the PIV measurements).

others for a rotating flat lid. However, as the space available at the central axis is smaller in the present case, the strength of the breakdown is also reduced for the same values of the governing parameters. This is in agreement with the findings of Yu et al. [13], who used a conical stationary lid to inhibit the occurrence of vortex breakdown. Altogether, this fundamentally leads to a shift of the main curve (single breakdown) in the stability diagram to larger values of $H / R$ and $R e$, as already shown in Fig. (2).

Although whole-field quantification has been carried out with the PIV system, the double breakdown has still been captured with detail. The remaining flow features, namely the global recirculation pattern and the boundary layer over the rotating cone are evidenced as well. Vorticity contours normalized by $\Omega$ are also shown in the figure, as this quantity is of primary interest in a vortical flow. Following the conclusions of a previous study [16], the vorticity field $\zeta(r, x)$ has been directly computed from the PIV measurements using centred differences. Apart from those naturally occurring on the container walls, two main regions of concentrated vorticity are observed on top of the primary breakdown bubble. The vorticity maxima in these areas will be later used in a quantitative comparison with the numerical data.

In order to facilitate this task, the velocity field obtained by numerical simulation in a non-uniform mesh has been interpolated (Krigging method) to the same (uniform) mesh used in the PIV measurements.

Again, both velocity vectors and vorticity contours computed by centred differences are presented in Fig. (5) for a meridional plane. The results evidence the difficulties of
PIV to cope with large velocity gradients, especially those occurring in the vicinity of solid boundaries. Wall boundary layers could not be accurately captured using the foregoing experimental technique in whole-field measurements. However, apart from these regions and the strongly decelerated flow on top of the primary breakdown bubble, a remarkably good agreement between experiments and simulations was obtained, even in the more stringent case of the vorticity field. This observation may be confirmed quantitatively as well in Table $\mathbf{1}$, which provides a comparison between the values of local maxima of vorticity obtained with each technique.

A detailed comparison is also made for axial velocity profiles taken at several locations, as shown in Fig. (6). The shortcomings of PIV near the side walls (all profiles) and close to the tip of the rotating cone $(x / R=0.0)$ can again be seen in this figure. As mentioned before, the region on top of the primary breakdown bubble $(x / R=1.8)$ is also affected by the limited spatial resolution of PIV, exhibiting a clear underprediction of the axial velocity in the jet-like profile occurring at this location. Furthermore, the size of the secondary breakdown bubble seems to be slightly smaller than in the numerical predictions, whereas the size of the primary one looks slightly larger.

A similar comparison is made in Fig. (7) between LDV measurements and, again, numerical simulations. The consequences of the better spatial accuracy displayed by this experimental technique is evidenced in the aforementioned jet-like profile and in the vicinity of side walls and cone. However, unexpected difficulties were sometimes encountered in the measurement of the peak velocities in the 
side wall boundary layers. A slight asymmetry is also seen in the experiments (both PIV and LDV), which reveals the sensitivity of the flow structures to a possibly imperfect alignment of the top wall $(x / R=2.0)$.

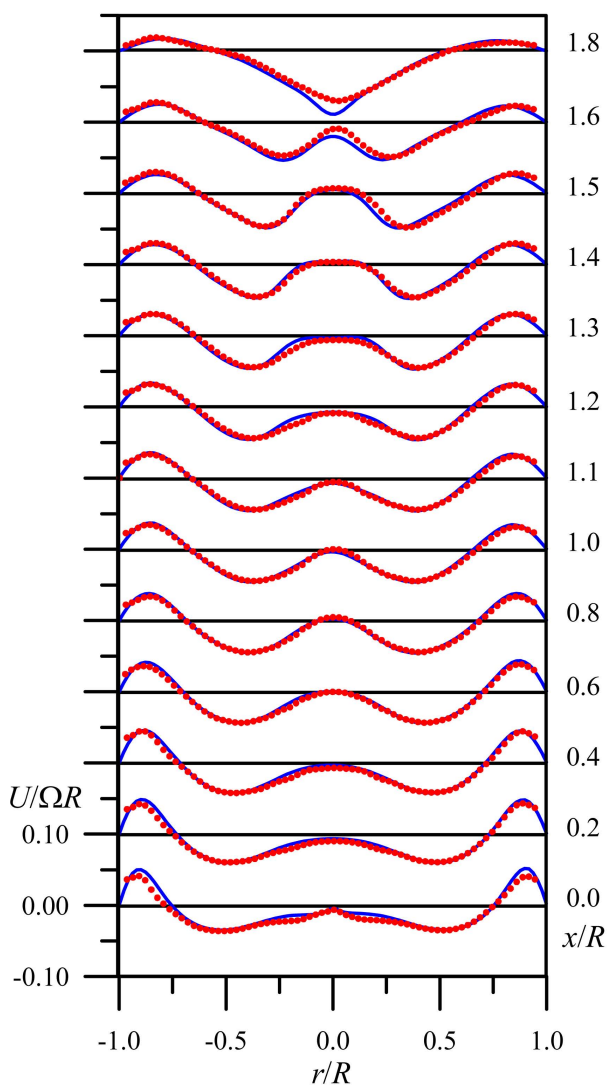

Fig. (6). Profiles of the axial velocity component: comparison between numerical simulations (blue lines) and PIV measurements (red symbols).

Table 1. Local Maxima of Vorticity at Reference Locations from Numerical Simulations and PIV

\begin{tabular}{|c|c|c|c|c|}
\hline $\boldsymbol{x} / \boldsymbol{R}$ & $\boldsymbol{r} / \boldsymbol{R}$ & $\begin{array}{c}\text { Numerical } \\
\text { (no interp.) }\end{array}$ & $\begin{array}{c}\text { Numerical } \\
\text { (interpol.) }\end{array}$ & PIV \\
\hline \hline 1.5 & 0.21 & -0.52 & -0.51 & -0.51 \\
\hline 1.8 & 0.23 & -0.50 & -0.50 & -0.46 \\
\hline
\end{tabular}

\section{CONCLUSIONS}

Numerical simulations of swirling flow inside a closed container have been carried out in the presence of steady vortex breakdown. These data have been used as a reference to assess the capabilities of PIV and LDV techniques for the characterisation of the associated flow topology. Flow maps embracing both axial/radial velocities and corresponding vorticity at a meridional plane have been compared in the case of numerical simulations and PIV. Several axial velocity profiles have also allowed a detailed comparison between experiments and simulations.

The results have evidenced that accurate measurements can be obtained with both PIV and LDV for a difficult flow condition, involving a double breakdown in the limit of the steady regime. The limitations of the PIV technique with

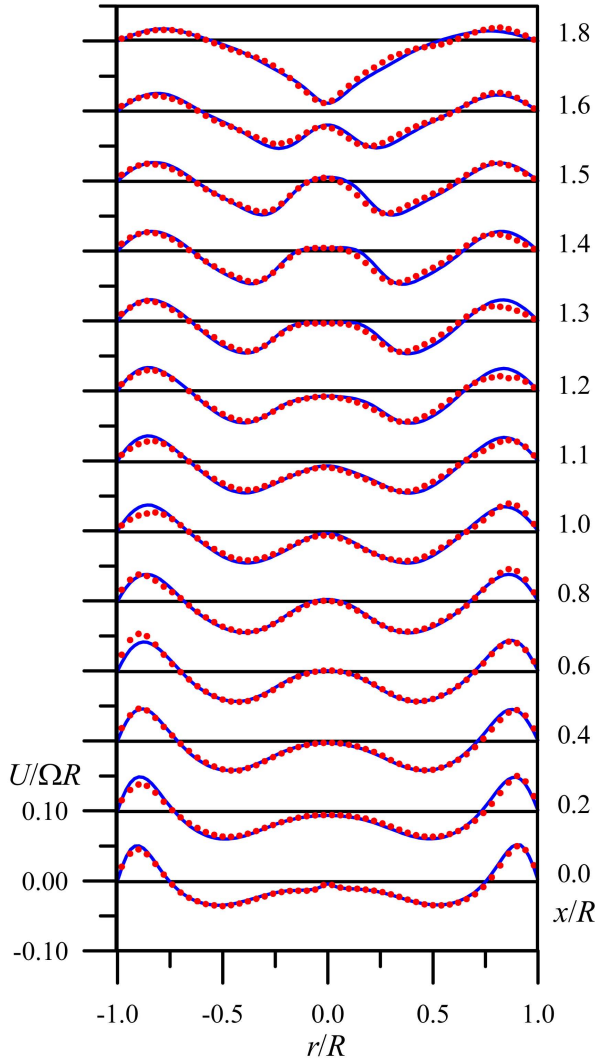

Fig. (7). Profiles of the axial velocity component: comparison between numerical simulations (blue lines) and LDV measurements (red symbols).

respect to spatial resolution have also been noted when a whole-field characterisation is performed. This mainly affects the description of wall boundary layers and the jetlike velocity region on top of the primary breakdown bubble. Better spatial resolution is naturally obtained with LDV at the expense of a much longer measurement time. However, a few inaccuracies have also been found with this technique in the quantification of the axial velocity maxima in the side wall boundary layers.

\section{REFERENCES}

[1] H. U. Vogel, "Experimentelle Ergebnisse über die Laminare Strömung in einem zylindrischen Gehäuse mit darin Rotierender Scheibe", Bericht 6, Max-Planck-Institut für Strömungsforschung, Göttingen, 1968

[2] M. P. Escudier, "Observations of the flow produced in a cylindrical container by a rotating endwall”, Exps. Fluids, vol. 2, pp. 189-196, December 1984.

[3] H. M. Blackburn and J. M. Lopez, "Symmetry breaking of the flow in a cylinder by a rotating endwall", Phys. Fluids, vol. 12, pp. 2698-2701, November 2000 .

[4] F. Sotiropoulos and Y. Ventikos, "The three-dimensional structure of confined swirling flows with vortex breakdown", J. Fluid Mech., vol. 426, pp. 155-175, January 2001

[5] A. Y. Gelfgat, P. Z. Bar-Yoseph, and A. Solan, "Three-dimensional instability of axisymmetric flow in rotating lid-cylinder enclosure", J. Fluid Mech., vol. 438, pp. 363-377, July 2001.

[6] F. Marques and J. M. Lopez, "Precessing vortex breakdown mode in an enclosed cylinder flow", Phys. Fluids, vol. 13, pp. 1679-1682, June 2001.

[7] E. Serre and P. Bontoux, "Vortex breakdown in a threedimensional swirling flow", J. Fluid Mech., vol. 459, pp. 347-370, May 2002. 
[8] J. M. Lopez, "Rotating and modulated rotating waves in transitions of an enclosed swirling flow", J. Fluid Mech., vol. 553, pp. 323346, April 2006

[9] C. H. Westergaard, P. Buchhave and J. N. Sørensen, "PIV measurements of turbulent and chaotic structures in a rotating flow using an optical correlator", in Laser Techniques and Applications in Fluid Mechanics, R. J. Adrian, D. F. G. Durão, F. Durst, T. Asanuma and J. H. Whitelaw, Eds. Berlin Heidelberg New York: Springer-Verlag, 1993, pp. 243-256.

[10] J. C. F. Pereira and J. M. M. Sousa, "Confined vortex breakdown generated by a rotating cone", J. Fluid Mech., vol. 385, pp. 287323, April 1999.

[11] I. V. Naumov, V. L. Okulov, K. E. Meyer, J. N. Sørensen and W. Z. Shen, "The LDA - PIV diagnostics and 3D simulation of oscillating swirl flow in a closed cylindrical container", Thermoph. Aeromech., vol. 10, pp. 143-148, May 2003.

[12] J. N. Sørensen, I. Naumov and R. Mikkelsen, "Experimental investigation of three-dimensional flow instabilities in a rotating lid-driven cavity", Exps. Fluids, vol. 41, pp. 425-440, September 2006.
[13] P. Yu, T. S. Lee, Y. Zeng and H. T. Low, "Effects of conical lids on vortex breakdown in an enclosed cylindrical chamber", Phys. Fluids, vol. 41, pp. 117101-1-9, November 2006.

[14] C. Freek, J. M. M. Sousa, W. Wentschel, and W. Merzkirch, "On the accuracy of an MJPEG-based digital image com-pression PIVsystem", Exps. Fluids, vol. 27, pp. 310-320, September 1999.

[15] H.-E. Albrecht, M. Borys, N. Damaschke, and C. Tropea, Laser Doppler and phase Doppler measurement techniques. Berlin Heidelberg New York: Springer-Verlag, 2002.

[16] J. M. M. Sousa and C. Freek, "On the advantages and pitfalls of statistical PIV analysis in the measurement of confined vortex breakdown", in Ninth International Symposium on Application of Laser Techniques to Fluid Mechanics, 1998, pp. 4.4.1-4.4.8.

[17] M. Raffel, C. Willert, and J. Kompenhans, Particle image velocimetry. Berlin Heidelberg New York: Springer-Verlag, 1998.

[18] J. C. F. Pereira, "Refractive index matching for LDV measurements near walls and in complex geometries", in Instrumentation for Combustion and Flow in Engines, D. F. G. Durão, J. H. Whitelaw, and P. O. Witze, Eds. Dordrecht: Kluwer Academic, 1988, pp. 267-284.

(C) J.M.M. Sousa; Licensee Bentham Open.

This is an open access article distributed under the terms of the Creative Commons Attribution License (http://creativecommons.org/licenses/by/2.5/), which permits unrestrictive use, distribution, and reproduction in any medium, provided the original work is properly cited. 